\title{
Incidence of Chronic Kidney Disease and Its Association with Fasting Blood Sugar Change Among Diabetic Patients in Resource Limited Setting in Case of Ethiopia: Bayesian Joint Modeling
}

Gebiso Roba Debele ( $\square$ gebisa.roba123@gmail.com )

Mettu University https://orcid.org/0000-0002-9788-5022

Haileab Fekadu Wolde

University of Gondar College of Medicine and Health Sciences

Melaku Kindie Yenit

University of Gondar College of Medicine and Health Sciences

Research article

Keywords: chronic kidney disease, fasting blood sugar, diabetes mellitus, Bayesian joint modeling

Posted Date: November 13th, 2020

DOI: https://doi.org/10.21203/rs.3.rs-104766/v1

License: (c) (i) This work is licensed under a Creative Commons Attribution 4.0 International License.

Read Full License 


\section{Abstract}

Background: Chronic kidney disease (CKD) is one of most common microvascular complication of diabetes and become a major health burden worldwide. Despite the effect of fasting blood sugar on the rate of prevent diabetic kidney disease has a pivotal role on the prevention of the diseases, their association is not well investigated. Therefore, the aim of this study was to assess Incidence and predictors of chronic kidney disease and its association with longitudinal fasting blood sugar change among diabetic patients in Southwest Ethiopia.

Methods: A retrospective follow-up study was conducted among type 1 and type 2 diabetes patients (DM) at Jimma university medical center, Southwest Ethiopia. A total of 405 diabetes patients who newly enrolled in the DM chronic care from 2012 to 2015 were selected using the simple random sampling technique. Selected patients with complete information were followed until February 2020. Data was extracted from patients' medical records. Cox proportional hazard with linear mixed effect model was jointly modeled using Bayesian approach, and $95 \%$ credible interval was used to select significant variables. Model adequacy was checked by residuals plot.

Results: A total of 405 DM patients with complete information were followed for 27494.44 person-month (PM) observation. Overall, 63 patients developed chronic kidney disease with an incidence rate of 2.29 per1000 PM observation (95\% Cl: [1.79, 2.93]. Time dependent fasting blood sugar was significantly associated with risk of CKD (AHR=4.23, 95\% Crl [2.53, 8.23]). Female sex (AHR= 0.51, 95\% Crl: [0.27, 0.94]), positive proteinuria (AHR= 2.85, 95\% Crl: $[1.48,5.55])$, having hypertension (AHR= $2.31,95 \% \mathrm{Crl}$ : $[1.03,5.56])$ and $\mathrm{HDL}<40 \mathrm{mg} / \mathrm{dl}(\mathrm{AHR}=3.19,95 \% \mathrm{Crl}[1.73,5.98])$ were significant predictors of CKD.

Conclusion: Chronic kidney disease among diabetes patients continue to be a significant public health problem in Ethiopia. Longitudinal fasting blood sugar change had significant associations with risk of CKD and high FBS can be used as a signal to screen DM patients for CKD. Female sex, positive proteinuria, hypertension and $\mathrm{HDL}-\mathrm{C}<40 \mathrm{mg} / \mathrm{dl}$ were significant predictors of chronic kidney disease.

\section{Introduction}

Diabetes mellitus (DM) is the most prevalent metabolic syndrome, characterized by high levels of plasma glucose (1). Globally, there were 463 million (9.3\%) prevalence of DM and projected to increase to 700.2 million by 2045 according to 2019 International diabetic federation (IDF). The prevalence of DM in Ethiopia was reported as $4.3 \%$ (2). One of the clinical importance of DM is largely due to its association with the development of chronic kidney disease (CKD) (3), in which $30 \%$ of type 1 DM (T1DM) and $40 \%$ of type 2 DM (T2DM) patients develops chronic kidney disease (CKD) throughout their life (4).

Kidney disease is one of the microvascular complication of DM which ranked the 12th most common cause of death, accounting for 1.1 million deaths worldwide in 2015 and which indicates increment of death by 39.5\% between 2005 and 2015 (5). Globally the prevalence of CKD estimated to be ranged from $8-16 \%$ from which DM is the leading cause (6). Diabetic nephropathy were estimated to be $35.3 \%$ and 
29.7\% in sub-Saharan and eastern African countries, respectively (7). In Ethiopia, studies in northern and southern parts of the country shows $21.8 \%$ and $23.8 \%$ prevalence of CKD in diabetes, respectively $(8,9)$.

CKD is an increasing clinical and public health problem related with adverse health outcomes including end stage renal disease (ESRD), heart failure and high costs of treatment once they develop ESRD (10). Diabetes alone contributes to $12-55 \%$ of ESRD and its incidence is ten times as high in adults with diabetes (11). Due to high costs of treatment in developing countries most people with ESRD continue to die in spite of established treatment options (12). Nine of ten patients diagnosed with ESRD in Asia and more than $70 \%$ of ESRD in India die within a few months due to scarce resources to get therapy $(13,14)$. Only less than $3 \%$ of ESRD get access to renal replacement therapy in middle and eastern Africa (15).

Different studies from various parts of the world identified various factors associated with CKD among DM patients, but mainly by the clinical, physiological and sociodemographic variables. Among sociodemographic variables female sex (16) and age (17) were positively associated with risk of CKD. Unlike the above a study evidence from USA (18) and Ethiopia (19) showed as being male have high risk to develop CKD compared to female. Among physiologic and clinical factors, DM patients with proteinuria $>300 \mathrm{mg} / \mathrm{L}(17)$, higher triglycerides and lower high density lipoprotein cholesterol (HDL-C) were increased risk of CKD (19-22). Individuals who were hypertensive (22) and higher body mass index (BMI) $(19,22)$ at the start of treatment was related with higher risk of CKD compared with their counter parts. Regarding the association of blood glucose with CKD in DM patients, Poor glycemic control is associated with development of ESRD (23) and intensive blood glucose control early have a long-lasting favorable effect on the risk of CKD development (24).

Despite knowledge of clinical biomarkers like change in Fasting Blood Sugar (FBS) is used to prevent or reduce the progression of CKD through early identification (25), the strength of association of FBS and risk of CKD is little known among DM patients. In Ethiopia, few studies conducted on CKD didn't assess the effect of predictors on CKD independently which might under/overestimate the actual effect of predictors. Moreover, most of previous studies were based on separate analysis of time to CKD which results in inefficient and unprecise estimate in case of endogenous covariate. Thus, our study aimed to determine incidence and predictors of chronic kidney disease and modeling its association with fasting blood sugar change among diabetes mellitus patients in Jimma University medical center, Southwest Ethiopia.

\section{Methods And Materials}

\section{Study setting}

The study was conducted at Jimma University Medical center, which is the oldest public hospitals in Ethiopia established in 1930 E.C by Italian invaders for the service of their soldiers and located in Jimma zone, Oromia regional state, Southwest Ethiopia, which is about $352 \mathrm{k}$ meters (KM) from Addis Ababa. The hospital provides services for approximately 9,000 inpatient and 80,000 outpatient attendances a 
year, coming from the catchment population of about 15 million people. Besides other services, chronic disease follows-up service is being provided in separated outpatient department (OPD) and there is diabetic clinic separated from other chronic disease.

\section{Study population and design}

An institution based retrospective follow-up study was conducted among T1DM and T2DM patients at Jimma University medical center. All T1DM and T2DM patients who had follow-up at the hospital whose age is greater or equal to 15 were our source population. All newly diagnosed T1DM and T2DM patients who were enrolled between September 2012 and August 2015 were our study population and patients with two or more fasting blood sugar measurement was included in the study. Patients who already developed CKD at the start of study and patients with unknown date of DM and CKD diagnosis were excluded from the study. Our dependent variables was chronic kidney disease incidence and defined as abnormalities of kidney structure or function that marked by glomerular filtration rate (GFR) $<60 \mathrm{ml} / \mathrm{min} / 1.73 \mathrm{~m}^{2}$ ), that present for $>3$ months and determined based on the clinical decision of the physician from the patient's medical records (26). Time to CKD was the time gap in month between diagnoses of diabetes mellitus to development of CKD. Death, lost to follow up and being event free at the end of the study were considered as a censored. Lost to follow up was defined as absence of the patients from the follow up for one year or more successive year from the date they last visited the diabetic clinic (27). Diabetic retinopathy was defined as a microvascular complication of DM that diagnosed by ophthalmoscopy or/and clinical examination by retinal specialists and then confirmed by fundus photography (28). WHO defined BMI as a physiological factors that measured by weight in kilograms per square of height in meters $\left(\mathrm{kg} / \mathrm{m}^{2}\right)$ and it is subdivided as low $\left(<18.5 \mathrm{~kg} / \mathrm{m}^{2}\right)$, normal $\left(18.5-24.9 \mathrm{~kg} / \mathrm{m}^{2}\right)$ and high $\left(\geq 25 \mathrm{~kg} / \mathrm{m}^{2}\right)(29)$. Hypertension (HTN) was an average of the first two systolic/diastolic blood pressure of $140 / 90 \mathrm{mmHg}$ or higher that taken on two or more separate days (30). In every follow up we assessed patients who were included in the study for chronic kidney disease and a baseline characteristic were taken from the patient's registration record at the start of study. Accordingly, socio demographic, clinical, comorbidities and physiologic characteristics was assessed registration document. All lipid profile (HDL-C, LDL-C, triglyceride and total cholesterol) was categorized for analysis based on American Association for Clinical Chemistry, mayo clinic and guideline from the National Cholesterol Education Program (31-33). Consecutively, a time varying endogenous covariate (repeatedly measured FBS in $\mathrm{mg} / \mathrm{dl}$ ) was assessed every four months from the start to the end of the study.

\section{Sample size determination and sampling procedure}

The sample size was determined using Schoenfeld formula for survival parts (34) by taking three factors significantly associated with risk of CKD from previous studies and using Diggle formula for repeated measurements or longitudinal parts (35). The following assumptions were considered to get the final sample size; $95 \%$ confidence level, $80 \%$ power and $10 \%$ of withdrawal probability. Accordingly, a final sample size of 432 was obtained. Study subjects were selected using simple random sampling 
technique. Sampling frame was prepared by collecting the identification number of DM patients from the registration book.

\section{Data collection procedures and quality control}

Secondary data were collected from patient intake form and follow-up that are recorded routinely by the hospitals for follow up, monitoring and evaluation of patients. Before actual data collection, preliminary review was conducted among $5 \%$ of sample size to evaluate the adequacy of checklist. Variables such as family history of renal problem, behavioral factors, occupation and adherence to treatment are not available in the record and not considered in this study. Data were collected using uniform checklists by health officers and nurses working in the hospitals. Filled checklist was checked for consistency and completeness before entry. Then data entry was cross-checked and we clarified the missing data.

\section{Data management and statistical analysis}

Data entry was done using Epi-Data version 4.6 and then exported to R statistical software 3.6.1 for further data cleaning and analysis. In order to avoid misspecification of missing data mechanism, we applied sensitivity analysis to multiple imputations and complete case analysis approach of missing data handling mechanism. The result showed there is no difference in parameter estimates under two methods. Complete case analysis was selected based on deviance information criteria (DIC) compared with imputed model. For continuous variables, mean with standard deviation (SD) for normally distributed and median with interquartile range (IQR) for non-normally distributed was used to describe the population. Categorical variables were described in terms of frequencies and percentages.

The cumulative incidence of CKD was calculated by taking the number of new chronic kidney disease as the numerator and the total initial population as the denominator. Patient-months at risk of developing CKD were calculated from the baseline appointment date to either the date of events or censoring. Accordingly, Incidence density was computed as the number of new cases divided by patient-months at risk.

Time to CKD was estimated using Kaplan-Meier (KM) and Log-rank test to compare survival time between groups of categorical variables. Before fitting linear mixed model for longitudinal sub-model, exploratory data analysis was done using individual and mean profile plots. Normality of longitudinal FBS was assessed using QQ-plot and normally distributed after log transformation.

Proportional hazard assumptions (PHA) were checked by cumulative log hazard plot and schoenfeld residuals method and all results supports the proportional hazards assumptions. Variables with a $p$ values less than 0.2 from the uni-variable analysis were included in the multivariable survival and linear mixed effect models. Best fitting survival (cox) and longitudinal (model with intercept and random slopes) sub model was selected based on Akakie Information Criterion (AIC) and further examined in Bayesian joint model. Then data was first analyzed using linear mixed effect model and cox regression model separately using $\mathrm{R}$ software. 
Bayesian joint models with different parameterizations were fitted using JMBayes package of $R$ software. Bayesian inference is based on the full posterior distribution in joint model like any Bayesian model. The expression for the posterior distribution of the model parameters is derived under the assumptions that given the random effects, both the longitudinal and event time process was independent, and the longitudinal responses of each subject are assumed independent. The full posterior distribution is derived from Likelihood and prior. In the current study we used a vague or flat prior distribution in that we selected priors and hyper parameter values in such a way that, the priors have minimal impact relative to the data. For our study, the standard prior distribution which was aided by Rigopoulos (36) for joint modeling in the JM Bayes package was considered. So, we used normal distribution prior (precision of 0.001 and mean zero) for fixed effect of longitudinal sub-model, regression coefficients of survival sub-model, association parameter and spline coefficients of the baseline risk function. We used gamma prior $(1,0.005)$ for precision parameter of survival and longitudinal sub model. The Wishart prior distribution was used for covariance matrix of random effects.

For model selection, we used DIC, a hierarchical modeling generalization of the AIC. Based on DIC comparison, the model with lagged value and slope parameterizations was identified to be an efficient model. To account for the effect of an endogenous time-varying covariate (FBS) on the hazard of CKD, we used the true unobserved value of FBS in the survival model. Association parameter (alpha value) from fitted Bayesian joint model was used to assess strength of association between longitudinal FBS change and CKD. In multivariable Bayesian joint model, a covariate with posterior mean that contained in the $95 \%$ credible interval was considered significant factors.

Time series plot was used assess convergence of chain because from the two schools of running Markov Chain Monte Carlo (MCMC) algorithms, namely the one long chain and multiple shorter chains school, JMBayes implements the former. It is a simple method of assessing chain convergence by looking at the history of iterations. If the chains show a reasonable degree of randomness between iterations, it signifies that the Markov chain has found an area of high likelihood and is integrating over the target density and hence indicating that it has converged. The goodness of fitness of the Bayesian joint model was assessed by using martingale residual for survival sub model and standardized marginal residual for longitudinal sub model.

\section{Results}

\section{Baseline characteristics of study participants}

A total of 405 newly diagnosed DM patients' cards were reviewed in the study with a response rate of $96.7 \%$. Thirty-two records with incomplete information, such as date of enrollment, outcome of interest and follow-up date, were excluded.

The mean (SD) of age of study participants at the start of the study was 43.9 (15.8) year and male constituted $61.5 \%$ and $242(59.8 \%)$ were urban residents. Nearly three-fourth $(70.4 \%)$ of patients had type- 
II DM. A quarter of respondents, 112 (27.7\%), had family history of DM. More than half 217 (53.6) of DM patients were on Oral Hypoglycemic Agent (OHA) treatment AKI and positive proteinuria were found among $22 \%$ and $22.2 \%$ of the study participants, respectively. About $66(16.3 \%)$ and $111(27.4 \%)$ of the patients had high total cholesterol and triglyceride level, respectively. More than half of the patients (57.8\%) had LDL-C level less than $100 \mathrm{mg} / \mathrm{dl}$ and $94(23.2 \%$ ) of study participants had HDL-C level less than $40 \mathrm{mg} / \mathrm{dl}$. and the median (IQR) of creatinine was $0.8(0.63,0.98) \mathrm{mg} / \mathrm{dl}$. One hundred twenty-two $(30.1 \%)$ of patients were obese with BMI greater than or equal to $25 \mathrm{~kg} / \mathrm{m}^{2}$. Regarding the comorbidities: 195(48.1\%), 81(20\%) and 55(13.6\%) of the participants had HTN, diabetic retinopathy and history of CHD, respectively. The mean (SD) of SBP was found to be 125.8(19.8) and median (IQR) for DBP was 80(70, 90) (Table:1).

\section{Chronic kidney disease in T1DM and T2DM}

The study participants were followed for a median follow up time of 70 months [IQR $=60.2-83.2)]$. Out of 405 study participants followed for a maximum of 89.8 months, 63 (15.56\%), 95\% Cl [12.24, 19.54] patients had developed CKD in 27494.44 PM of observations. The incidence density was 2.29/1000 PM with $95 \% \mathrm{Cl}$ of $[1.79,2.93]$ or 2.75 per 100 - person year (PY) $95 \% \mathrm{Cl}$ of $[2.15,3.52]$. Survival probability was decreases as follow-up increases (Fig. 1). The cumulative survival probability of patients was 0.975 at 20 months, 0.937 at 40 months, 0.867 at 60 months and 0.781 by the end of the study.

\section{Exploring longitudinal fasting blood sugar change}

A minimum of three and a maximum of twenty-three fasting blood sugar measurement were taken during the study period. This means, data were unbalanced and there was different number of measurements per subject. Individual profile plots show there is high variability of FBS within and between patients. A different starting point suggests random intercept model and non-constancy of trajectory of FBS over time for patients suggests considering mixed model with random slope (Fig. 2). The mean profile plots of FBS level for diabetic patients are nonlinear and imposing linear mixed model may be too restrictive and yield unsatisfactory results. Due to this, we used natural cubic spline to longitudinal model which handle the correlation that is not handled by random effects and yield satisfactory results. The mean of FBS among DM patients who had CKD was higher than those who did not develop CKD and this supports application of joint modeling on this data (Fig. 3).

\section{Bayesian Joint Models}

Cox proportional hazard and linear mixed effect (using penalized spline) model was selected as a better model because of lowest AIC. Bayesian joint models were fitted using lagged effect of both current value and slope parameterizations under MCMC to express the correct relationship between the change in FBS and risk of CKD. This lagged effect model was selected by DIC value from different parameterizations. Therefore, a Bayesian joint model of cox proportional hazard and linear mixed effect (using penalized spline) model with lagged value and slope parameterization was the final model. 
Bayesian joint model was converged after we used 20 numbers of knots, 10 thinning, 50,000 burning in period and 400,000 numbers of iterations. To assess the goodness of fitness of Bayesian joint model, we used standardized marginal residuals for the longitudinal and martingale residual for survival sub model. We observe that the fitted loess curve (blue color) in the plot of the standardized marginal residuals versus the fitted values shows no systematic trend with residuals which indicates goodness of fitness was satisfied. From the martingale residual we observe that for fitted values there is no deviation of the loess smoother from zero (Fig. 4). Therefore, survival sub model of Bayesian joint modeling also satisfied the goodness of fitness.

\section{Predictors of CKD and its association with FBS change}

The multivariable Bayesian joint analysis was fitted using the Cox-Regression and linear mixed model of longitudinal FBS change. Covariates like sex, hypertension status, proteinuria at baseline and HDL-C level were found to be independent predictors for CKD among T1DM and T2DM patients. Longitudinal fasting blood sugar change was strongly associated with the risk of CKD (Table 2). A 50\% increase of fasting blood sugar, increases the risk of developing chronic kidney disease among T1DM and T2DM patients by 4.23 , fold. The hazard of experiencing CKD was decreased by almost half among female patients as compared to male patients. The risk of CKD was 2.85 times higher among DM patients with positive protein urea as compared to negative protein urea. The risk of CKD for patients who have hypertension at baseline was 2.31 times higher than that of patients who have no hypertension. After adjusting for other variables in the model the hazard of experiencing CKD was 3.19 times higher for DM patients with HDL-C $<40 \mathrm{mg} / \mathrm{dl}$ as compared to those with HDL-C > $=40 \mathrm{mg} / \mathrm{dl}$. Since our main aim was survival components, the longitudinal sub model was summarized in Table 3 .

\section{Discussion}

This current study investigated the incidence and predictors of chronic kidney disease as well as effects of FBS change on risk of CKD among T1DM and T2DM patients at Jimma university medical center, Southwest Ethiopia. Longitudinal FBS change was strongly associated with the risk of CKD. Factors like: sex, proteinuria, HTN and HDL-C were found to be significantly associated with risk of CKD.

In our study, $15.56 \%$ (cumulative incidence) of the study participants had developed CKD with the incidence density of 2.291 per 1000 PM or 2.75 per 100 -PY of observation. This study revealed a higher incidence of CKD than the studies done in Korea 12.1\% (37) and Netherland 12.18 per 1000PY (38).

These differences could be because of the age of onset of T2DM is became decreasing, allowing patients enough duration of diabetes to develop CKD (39). Moreover, the black race was associated with a greater rate of GFR decline (40) and this might be due to the presence of apolipoprotein L1 high-risk genotype for renal impairment in black race (41).

However, incidence of CKD in the current study was lower than studies done in Australia 7.4 per 100PY (42), Morocco $34.7 \%$ (43) and UK with $29 \%$ of cumulative incidence (44). This could be due to the higher life expectancy of Australian (83.9 year), UK (81.7 year) and Morocco (77.4 year) population compared to Ethiopian (67.7 year) population (45). After the age of 30 years, GFR progressively declines at an average 
rate of $8 \mathrm{ml} / \mathrm{min} / 1.73 \mathrm{~m} 2$ per decade (46). This might be due to structural and functional changes of kidney in older patients $(47,48)$.

In the current study, we observed that the longitudinal FBS change was strongly associated with the risk for the chronic kidney disease. Though there is no study which assess strength of association of longitudinal FBS change with risk CKD among T1DM and T2DM patients, there are studies that show as blood glucose is highly related with CKD in DM patients. A randomized study in individuals with T1DM have shown that control of blood glucose through intensive treatment slows progression of microvascular complications (nephropathy) and reduces the development and progression of microalbuminuria (49). Other study shows the strong positive association between glycated hemoglobin (HbA1c) concentration which is highly correlated with FBS and incident CKD (50). This might be due to damage of renal vessels, nerves of bladder and urinary tract by high blood glucose in diabetes. Gradually, high blood sugar levels can cause renal blood insufficiency due to clotting. Pressure from a full bladder can damage kidneys because fail to feel full bladder due bladder nerve damage. long time urine accumulation in bladder, may result in urinary tract infection because high sugar level is favorable for the growth of bacteria and this infection can spread to kidneys (51).

In our study female was found to have lower risk of developing CKD as compared to male patients. These finding is congruent with prospective study in USA (18) and study done by Ravid et al. (52). The reason might be due to estrogen, the hormone that is absent in male, which can improve glycemic control and lipid metabolism and eventually decrease the risk of CKD in DM patients (53). Estrogen from the onset of diabetes can reduce albuminuria, glomerulosclerosis, and tubulointerstitial fibrosis, suggesting that estrogen may be reno-protective $(54,55)$. However result of the current study is incongruent with studies done in other parts of USA (16) and Spain (17). Therefore, taking female sex as a protective or risk for CKD in DM patient would be difficult and indicates there is a need of further investigation about this issue.

In addition, positive proteinuria increases risk of having CKD as compared to negative proteinuria. This is in agreement with studies done in Ethiopia (27), Spain (17) and Japan (56). This might be due the crucial role of proteinuria in accelerating kidney disease progression to ESRD, promoting loss of kidney function and scarring through induction of tubular chemokine expression and intrarenal activation of complement cascade that cause renal injury through formation of membrane attack complex and possibly other biologically active products that interact with specific receptors $(57,58)$.

According to our finding, the hazard of experiencing CKD is much higher among DM patients who had HTN compared to their counterparts. This result is consistent with previous studies in Ethiopia (27), Spain (17) and china (22). The possible reason could be the HTN can affect endothelial cell structure and functioning that leads to enhanced abnormal growth of endothelial cell and vasoconstriction. This changes to the endothelium leads to development of glomerulosclerosis which predispose patients for CKD (59). 
Patients with $<40 \mathrm{mg} / \mathrm{dl} \mathrm{HDL-C}$ had higher risks of developing CKD compared their counterparts. This result was supported by studies conducted in Ethiopia (27) and other studies done by Munter P. et al. (20) and Bowe B. et al. (21). Biologically, HDL-C transports fats (lipids) away from the artery wall to the liver. This eventually reduces accumulation of fats and arthrosclerosis within the arterial wall and at the same time it protects the inner wall of the arteries from damage so this reduces the risk of vascular complication of DM including CKD (60). Therefore, patients with low level of HDL-C might lack this function of HDL-C and became at higher risk of CKD.

The finding of this study was intended to provide information for health professionals as high FBS can be used as a signal to screen DM patients for CKD. In addition, it provides information about factors associated with the risk of CKD and to act on them to minimize the risk and maximize their effort on prevention. The public health importance of the finding of this study is to reduce economic forfeiture related to dialysis and transplantation from ESRD.

Though our study has strength to estimate the incidence of CKD and its predictors using advanced modeling, it is not free from limitations. The nature of the study limits to know the exact time of onset of DM and CKD; as a result, it is difficult to determine how long an individual delayed to follow up. In addition, the retrospective nature of the method prevented the inclusion of all possible predictors that affect CKD. The study assumed all the chronic kidney disease are caused by diabetes mellitus and may overestimate the rate of CKD. In our study area HbA1c were not done for follow-up which is the recommended mode of testing blood sugar control, leading to the use of fasting blood sugar. Despite, of these limitations, this study determined incidence and predictors of CKD as well as modelled its association with FBS change among T1DM and T2DM patients using Bayesian joint model.

\section{Conclusion}

In the current study, incidence of CKD was relatively high compared with previous similar study in Ethiopia and influenced by multiple factors. Unobserved true lagged value of fasting blood sugar change had strong significant associations with risk of CKD. Female sex, positive proteinuria, having hypertension and HDL-C $<40 \mathrm{mg} / \mathrm{dl}$ were significant predictors of incident CKD. In light of these findings, we recommend health professionals to strength the routine monitoring of FBS as a biomarker which provides meaningful prognostic information that can help to differentiate patients with regard CKD and prevents its progression to ESRD. Health professionals should give greater attention to T1DM and T2DM patients with proteinuria, $\mathrm{HDL}-\mathrm{C}<40 \mathrm{mg} / \mathrm{dl}$ and hypertension together with that of $\mathrm{DM}$.

\section{Abbreviations}

AHR: Adjusted Hazard Ratio, AKI: Acute Kidney Injury, BMI: Body Mass Index, Cl/Crl: Confidence/Credible, CHR: Crude Hazard Ratio, Interval, CKD: Chronic Kidney Disease, CHD: Cardiac Heart Disease, DBP: Diastolic Blood Pressure, DIC: Deviance Information Criteria, DM: Diabetes Mellitus, ESRD: End Stage Renal Disease, FBS: Fasting Blood Sugar, GFR: Glomerular Filtration Rate, HbA1c: Glycoceted 
Hemoglobin, HDL-C: High-Density Lipoprotein Cholesterol, HTN: Hypertension, $\mathrm{Kg} / \mathrm{m}^{2}: \mathrm{Kilogram}$ per Square Meters, KM: Kaplan Meier, LDL-C: Low-Density Lipoprotein Cholesterol, MCMC: Markov Chain Monte Carlo, OHA: Oral Hypoglycemic Agent, PHA: Proportional Hazard Assumption, PY: Person Years, SBP: Systolic Blood Pressure, T1DM: Type One Diabetes Mellitus, T2DM: Type Two Diabetes Mellitus, USA: United States of America

\section{Declarations}

\section{Ethical approval and consent to participate}

Ethical clearance was obtained from the Institutional Review Board of the University of Gondar. Letter of cooperation was also obtained from Jimma university medical center. Permission letter was obtained from medical director of the hospitals in order to access medical records of patients. Data confidentiality was kept during all phases of research activities and held on secured password protected system.

\section{Consent for publication}

Not applicable

\section{Availability of data and materials}

Data will be available from the corresponding author upon request.

\section{Competing interests}

There is no competing of interests related to this work

\section{Funding}

The University of Gondar has covered the costs of data collectors and supervisors per diem. However, the University had no role in study design, data collections, and analysis, decision to publish, or preparation of the manuscript.

\section{Authors' contributions}

GRD designed the study, supervised data collection, performed data analysis and interpretation, and drafted the manuscript. HFW assisted in designing the study, did data analysis and interpretation, and critical review the manuscript. MKY assisted in designing the study, did data analysis and interpretation, and revised the manuscript. All authors read and approved the final manuscript and agree to be accountable for all the contents of the work in the manuscript.

\section{Acknowledgments}


Our heartfelt gratitude goes to the University of Gondar, College of Health and Medical Sciences for support by all necessary services. Additionally, we appreciate the support from Hospitals administrations and data collector.

\section{References}

1. WHO. Definition, diagnosis and classification of diabetes mellitus and its complications: report of a WHO consultation. Geneva; 1999.

2. Inernational Diabetes federation DIABETES ATLAS, Ninth edition 2019.

3. Rao Kondapally Seshasai S, Kaptoge S, Thompson A, Di Angelantonio E, Gao P, Sarwar N, et al. Diabetes mellitus, fasting glucose, and risk of cause-specific death. N Engl J Med. 2011;364(9):82941.

4. Reutens AT. Epidemiology of diabetic kidney disease. Medical Clinics. 2013;97(1):1-18.

5. Wang H, Naghavi M, Allen C, Barber RM, Bhutta ZA, Carter A, et al. Global, regional, and national life expectancy, all-cause mortality, and cause-specific mortality for 249 causes of death, 1980-2015: a systematic analysis for the Global Burden of Disease Study 2015. The lancet. 2016;388(10053):1459-544.

6. Jha V, Garcia-Garcia G, Iseki K, Li Z, Naicker S, Plattner B, et al. Chronic kidney disease: global dimension and perspectives. The Lancet. 2013;382(9888):260-72.

7. Wagnew F, Eshetie S, Kibret GD, Zegeye A, Dessie G, Mulugeta H, et al. Diabetic nephropathy and hypertension in diabetes patients of sub-Saharan countries: a systematic review and meta-analysis. BMC research notes. 2018;11(1):565.

8. Fiseha T, Kassim M, Yemane T. Prevalence of chronic kidney disease and associated risk factors among diabetic patients in southern Ethiopia. Am J Health Res. 2014;2(4):216-21.

9. Damtie S, Biadgo B, Baynes HW, Ambachew S, Melak T, Asmelash D, et al. Chronic Kidney Disease and Associated Risk Factors Assessment among Diabetes Mellitus Patients at A Tertiary Hospital, Northwest Ethiopia. Ethiopian journal of health sciences. 2018;28(6).

10. Go A, Chertow G, Fan D. Chronic kidney disease and the risks of death, cardiovascular events, and hospitalization. Journal of Vascular Surgery. 2005;41(1):177.

11. Roglic G. WHO Global report on diabetes: A summary. International Journal of Noncommunicable Diseases. 2016;1(1):3.

12. Neuen BL, Chadban SJ, Demaio AR, Johnson DW, Perkovic V. Chronic kidney disease and the global NCDs agenda. BMJ Specialist Journals; 2017.

13. Abraham G. The challenges of renal replacement therapy in Asia. Nature Publishing Group; 2008.

14. Rajapurkar MM, John GT, Kirpalani AL, Abraham G, Agarwal SK, Almeida AF, et al. What do we know about chronic kidney disease in India: first report of the Indian CKD registry. BMC nephrology. 2012;13(1):10. 
15. Liyanage T, Ninomiya T, Jha V, Neal B, Patrice HM, Okpechi I, et al. Worldwide access to treatment for end-stage kidney disease: a systematic review. The Lancet. 2015;385(9981):1975-82.

16. Yu MK, Katon W, Young BA. Associations between sex and incident chronic kidney disease in a prospective diabetic cohort. Nephrology. 2015;20(7):451-8.

17. Salinero-Fort MA, San Andrés-Rebollo FJ, de Burgos-Lunar C, Gómez-Campelo P, Chico-Moraleja RM, de Andrés AL, et al. Five-year incidence of chronic kidney disease (stage 3-5) and associated risk factors in a Spanish cohort: the MADIABETES Study. PLoS One. 2015;10(4):e0122030.

18. Lipworth L, Mumma MT, Cavanaugh KL, Edwards TL, Ikizler TA, Tarone RE, et al. Incidence and predictors of end stage renal disease among low-income blacks and whites. PLoS One. 2012;7(10):e48407.

19. Geletu AH, Teferra AS, Sisay MM, Teshome DF. Incidence and predictors of chronic kidney diseases among type 2 diabetes mellitus patients at St. Paul's Hospital, Addis Ababa, Ethiopia. BMC Res Notes. 2018;11(1):532.

20. Muntner P, Coresh J, Smith JC, Eckfeldt J, Klag MJ. Plasma lipids and risk of developing renal dysfunction: the atherosclerosis risk in communities study. Kidney Int. 2000;58(1):293-301.

21. Bowe B, Xie Y, Xian H, Balasubramanian S, Al-Aly Z. Low levels of high-density lipoprotein cholesterol increase the risk of incident kidney disease and its progression. Kidney international. 2016;89(4):88696.

22. Luk AOY, So WY, Ma RCW, Kong APS, Ozaki R, Ng VSW, et al. Metabolic Syndrome Predicts New Onset of Chronic Kidney Disease in 5,829 Patients With Type 2 Diabetes: A 5-year prospective analysis of the Hong Kong Diabetes Registry. Diabetes Care. 2008;31(12):2357-61.

23. Caramori ML, Parks A, Mauer M. Renal lesions predict progression of diabetic nephropathy in type 1 diabetes. Journal of the American Society of Nephrology. 2013;24(7):1175-81.

24. UKPDS G. Intensive blood-glucose control with sulphonylureas or insulin compared with conventional treatment and risk of complications in patients with type 2 diabetes (UKPDS 33). The lancet. 1998;352(9131):837-53.

25. Scirica BM. Use of biomarkers in predicting the onset, monitoring the progression, and risk stratification for patients with type 2 diabetes mellitus. Clinical chemistry. 2017;63(1):186-95.

26. Eknoyan G, Lameire N, Eckardt K, Kasiske B, Wheeler D, Levin A, et al. KDIGO 2012 clinical practice guideline for the evaluation and management of chronic kidney disease. Kidney Int. 2013;3(1):5-14.

27. Wolde HF, Atsedeweyen A, Jember A, Awoke T, Mequanent M, Tsegaye AT, et al. Predictors of vascular complications among type 2 diabetes mellitus patients at University of Gondar Referral Hospital: a retrospective follow-up study. BMC Endocr Disord. 2018;18(1):52.

28. Wilkinson CP, Ferris FL, 3rd, Klein RE, Lee PP, Agardh CD, Davis M, et al. Proposed international clinical diabetic retinopathy and diabetic macular edema disease severity scales. Ophthalmology. 2003;110(9):1677-82.

29. WHO. Physical status: the use and interpretation of anthropometry. Report of a WHO Expert Committee. Geneva;. World Health Organization technical report series. 1995;854:1-452. 
30. WHO. Diagnosis and management for patients with hypertension: Noncommunicable disease education manual for primary health care professionals and patients. 2017.

31. Grundy SM. Third report of the national cholesterol education program (NCEP) expert panel on detection, evaluation, and treatment of high blood cholesterol in adults (Adult Treatment Panel III) final report. Circulation. 2002;106:3143-421.

32. Cholesterol. Lab Tests Online. American Association for Clinical Chemistry. 2016 [Available from: https://labtestsonline.org/tests/cholesterol.

33. Cholesterol test. Mayo Clinic 2019 [Available from: https://www.mayoclinic.org/testsprocedures/cholesterol-test/about/pac-20384601.

34. Schoenfeld DA. Sample-size formula for the proportional-hazards regression model. Biometrics. 1983:499-503.

35. Diggle P, Diggle PJ, Heagerty P, Heagerty PJ, Liang K-Y, Zeger S. Analysis of longitudinal data: Oxford University Press; 2002.

36. Rizopoulos $\mathrm{D}$. The R package JMbayes for fitting joint models for longitudinal and time-to-event data using MCMC. arXiv preprint arXiv:14047625. 2014.

37. Kim WJ, Kim SS, Bae MJ, Yi YS, Jeon YK, Kim BH, et al. High-normal serum uric acid predicts the development of chronic kidney disease in patients with type 2 diabetes mellitus and preserved kidney function. J Diabetes Complications. 2014;28(2):130-4.

38. van Blijderveen JC, Straus SM, Zietse R, Stricker BH, Sturkenboom MC, Verhamme KM. A populationbased study on the prevalence and incidence of chronic kidney disease in the Netherlands. International urology and nephrology. 2014;46(3):583-92.

39. Koopman RJ, Mainous AG, Diaz VA, Geesey ME. Changes in age at diagnosis of type 2 diabetes mellitus in the United States, 1988 to 2000. The Annals of Family Medicine. 2005;3(1):60-3.

40. Hunsicker LG, Adler S, Caggiula A, England BK, Greene T, Kusek JW, et al. Predictors of the progression of renal disease in the Modification of Diet in Renal Disease Study. Kidney international. 1997;51(6):1908-19.

41. Grams ME, Rebholz CM, Chen Y, Rawlings AM, Estrella MM, Selvin E, et al. Race, APOL1 risk, and eGFR decline in the general population. Journal of the American Society of Nephrology. 2016;27(9):2842-50.

42. Schimke K, Chubb SA, Davis WA, Davis TM. Helicobacter pylori cytotoxin-associated gene-A antibodies do not predict complications or death in type 2 diabetes: the Fremantle Diabetes Study. Atherosclerosis. 2010;212(1):321-6.

43. Bentata Y, Haddiya I, Latrech H, Serraj K, Abouqal R. Progression of diabetic nephropathy, risk of endstage renal disease and mortality in patients with type-1 diabetes. Saudi Journal of Kidney Diseases and Transplantation. 2013;24(2):392.

44. Retnakaran R, Cull CA, Thorne KI, Adler Al, Holman RR. Risk factors for renal dysfunction in type 2 diabetes: UK Prospective Diabetes Study 74. Diabetes. 2006;55(6):1832-9. 
45. Worldometer. Life Expectancy of the World Population, Countries ranked by life expectancy 2020 [Available from: https://www.worldometers.info/demographics/life-expectancy/.

46. Coresh J, Astor BC, Greene T, Eknoyan G, Levey AS. Prevalence of chronic kidney disease and decreased kidney function in the adult US population: Third National Health and Nutrition Examination Survey. American journal of kidney diseases. 2003;41(1):1-12.

47. Hayslett JP, Kashgarian M, Epstein FH. Functional correlates of compensatory renal hypertrophy. The Journal of clinical investigation. 1968;47(4):774-82.

48. Thomson SC, Vallon V, Blantz RC. Kidney function in early diabetes: the tubular hypothesis of glomerular filtration. American Journal of Physiology-Renal Physiology. 2004;286(1):F8-F15.

49. Control TD, Group CDR. Effect of intensive therapy on the development and progression of diabetic nephropathy in the Diabetes Control and Complications Trial. Kidney International. 1995;47(6):170320.

50. Bash LD, Selvin E, Steffes M, Coresh J, Astor BC. Poor glycemic control in diabetes and the risk of incident chronic kidney disease even in the absence of albuminuria and retinopathy: Atherosclerosis Risk in Communities (ARIC) Study. Archives of internal medicine. 2008;168(22):2440-7.

51. NKF. Diabetes and Kidney Disease (Stages 1-4) 2014 [updated November, 2014. Available from: https://www.kidney.org/atoz/content/Diabetes-and-Kidney-Disease-Stages1-4.

52. Ravid M, Brosh D, Ravid-Safran D, Levy Z, Rachmani R. Main risk factors for nephropathy in type 2 diabetes mellitus are plasma cholesterol levels, mean blood pressure, and hyperglycemia. Archives of internal medicine. 1998;158(9):998-1004.

53. Ferrara A, Karter AJ, Ackerson LM, Liu JY, Selby JV. Hormone replacement therapy is associated with better glycemic control in women with type 2 diabetes: The Northern California Kaiser Permanente Diabetes Registry. Diabetes Care. 2001;24(7):1144-50.

54. Dixon A, Wells CC, Singh S, Babayan R, Maric C. Renoprotective effects of a selective estrogen receptor modulator, raloxifene, in an animal model of diabetic nephropathy. American journal of nephrology. 2007;27(2):120-8.

55. Mankhey RW, Wells CC, Bhatti F, Maric C. 17ß-Estradiol supplementation reduces tubulointerstitial fibrosis by increasing MMP activity in the diabetic kidney. American Journal of PhysiologyRegulatory, Integrative and Comparative Physiology. 2007;292(2):R769-R77.

56. Jiang G, Luk AOY, Tam CHT, Xie F, Carstensen B, Lau ESH, et al. Progression of diabetic kidney disease and trajectory of kidney function decline in Chinese patients with Type 2 diabetes. Kidney International. 2019;95(1):178-87.

57. Cravedi P, Remuzzi G. Pathophysiology of proteinuria and its value as an outcome measure in chronic kidney disease. British journal of clinical pharmacology. 2013;76(4):516-23.

58. Nangaku M, Pippin J, Couser WG. Complement membrane attack complex (C5b-9) mediates interstitial disease in experimental nephrotic syndrome. Journal of the American Society of Nephrology. 1999;10(11):2323-31. 
59. Hsueh WA, Anderson PW. Hypertension, the endothelial cell, and the vascular complications of diabetes mellitus. Hypertension. 1992;20(2):253-63.

60. Link JJ, Rohatgi A, de Lemos JA. HDL cholesterol: physiology, pathophysiology, and management. Current problems in cardiology. 2007;32(5):268-314.

\section{Tables}


Table 1

Socio-demographic, clinical, Physiologic characteristics of DM patients on treatment at Jimma university medical center, Southwest Ethiopia, 2012 to 2020.

\begin{tabular}{|c|c|c|c|}
\hline Variables & Censored $(n=342)$ & Event $=\operatorname{CKD}(n=63)$ & Total $(n=405)$ \\
\hline \multicolumn{4}{|l|}{ Sex } \\
\hline Male & 205(59.9) & $44(69.8)$ & $249(61.5)$ \\
\hline Female & $137(40.1)$ & 19(30.2) & 156(38.5) \\
\hline \multicolumn{4}{|l|}{ Residence } \\
\hline Urban & $208(60.8)$ & $33(52.4)$ & $242(59.8)$ \\
\hline Rural & 134(39.2) & $30(47.6)$ & $163(40.2)$ \\
\hline \multicolumn{4}{|c|}{ Family history of DM } \\
\hline No & 246(71.9) & $47(74.6)$ & 293(72.3) \\
\hline Yes & $96(28.1)$ & $16(25.4)$ & $112(27.7)$ \\
\hline \multicolumn{4}{|c|}{ Type of DM } \\
\hline T1DM & $110(32.2)$ & $10(15.9)$ & $120(29.6)$ \\
\hline T2DM & $232(67.8)$ & $53(84.1)$ & $285(70.4)$ \\
\hline \multicolumn{4}{|c|}{ Type of treatment } \\
\hline $\mathrm{OHA}$ & $176(51.5)$ & $41(65.1)$ & $217(53.6)$ \\
\hline Insulin & $111(32.5)$ & 11(17.5) & $122(30.1)$ \\
\hline Both & $55(16.1)$ & $11(17.5)$ & $66(16.3)$ \\
\hline \multicolumn{4}{|c|}{ History of AKI } \\
\hline No & 293(85.7) & $23(36.5)$ & $316(78.0)$ \\
\hline Yes & $49(14.3)$ & $40(63.5)$ & $89(22.0)$ \\
\hline \multicolumn{4}{|l|}{ Proteinuria } \\
\hline Negative & $296(86.5)$ & 19(30.2) & $315(77.8)$ \\
\hline Positive & $46(13.5)$ & $44(69.8)$ & $90(22.2)$ \\
\hline \multicolumn{4}{|c|}{ Total cholesterol(mg/dl) } \\
\hline Desirable & $227(66.4)$ & $23(36.5)$ & $250(61.7)$ \\
\hline Borderline & $58(17.0)$ & $16(25.4)$ & $74(18.3)$ \\
\hline High & $42(12.3)$ & $24(28.1)$ & $66(16.3)$ \\
\hline
\end{tabular}




\begin{tabular}{|c|c|c|c|}
\hline Variables & Censored $(n=342)$ & Event $=\operatorname{CKD}(n=63)$ & Total $(n=405)$ \\
\hline \multicolumn{4}{|c|}{ Triglyceride(md/dl) } \\
\hline Normal & $181(52.9)$ & 11(17.5) & 192(47.4) \\
\hline Borderline & $56(16.4)$ & $23(36.5)$ & $79(19.5)$ \\
\hline High & $82(24.0)$ & $29(46.0)$ & $111(27.4)$ \\
\hline \multicolumn{4}{|c|}{ LDL-C(md/dl) } \\
\hline$<100$ & $231(67.5)$ & 21(33.3) & $234(57.8)$ \\
\hline$>=100$ & 109(32.0) & $42(66.7)$ & $151(37.3)$ \\
\hline \multicolumn{4}{|c|}{$\mathrm{HDL}-\mathrm{C}(\mathrm{md} / \mathrm{dl})$} \\
\hline$>=40$ & $264(77.2)$ & $20(31.7)$ & $284(70.1)$ \\
\hline$<40$ & $51(14.9)$ & $43(68.3)$ & $94(23.2)$ \\
\hline \multicolumn{4}{|c|}{$\mathrm{BMI}\left(\mathrm{kg} / \mathrm{m}^{2}\right)$} \\
\hline$<18.5$ & $24(7.0)$ & $2(3.2)$ & $26(6.4)$ \\
\hline $18.5-24.9$ & $236(69.0)$ & 12(19.0) & $248(61.2)$ \\
\hline$>=25$ & $73(21.4)$ & 49(77.8) & $122(30.1)$ \\
\hline \multicolumn{4}{|c|}{ Hypertension } \\
\hline No & $200(58.5)$ & $10(15.9)$ & $210(51.9)$ \\
\hline Yes & $142(41.5)$ & $53(84.1)$ & 195(48.1) \\
\hline \multicolumn{4}{|c|}{ Diabetic retinopathy } \\
\hline No & 291(85.1) & $33(52.4)$ & $324(80.0)$ \\
\hline Yes & $51(14.9)$ & $30(47.6)$ & $81(20.0)$ \\
\hline \multicolumn{4}{|c|}{ Cardiac heart disease } \\
\hline No & $308(90.1)$ & $42(66.7)$ & $350(86.4)$ \\
\hline Yes & $34(9.9)$ & 21(33.3) & $55(13.6)$ \\
\hline Age & $42.7 \pm 15.37$ & $50.1 \pm 16.8$ & $43.9 \pm 15.8$ \\
\hline SBP & $123.99 \pm 18.92$ & $135.7 \pm 21.5$ & $125.8 \pm 19.8$ \\
\hline DBP & $80(70,90)$ & $85(70,90)$ & $80(70,90)$ \\
\hline Creatinine & $0.8(0.630 .98)$ & $2.3(1.8,3)$ & $0.85(0.67,1.2)$ \\
\hline
\end{tabular}


Table 2

Survival sub models with association parameter for DM patients on treatment at Jimma university medical center, Southwest Ethiopia, 2012 to 2020.

\begin{tabular}{|c|c|c|c|}
\hline Variables & Categories & CHR [95\% Crl] & AHR [95\% Crl] \\
\hline \multirow[t]{2}{*}{ Age } & $<65$ & 1 & 1 \\
\hline & $>=65$ & $1.97[1.01,3.72]$ & $1.26[0.61,2.49]$ \\
\hline \multirow[t]{2}{*}{ Sex } & Male & 1 & 1 \\
\hline & Female & $0.57[0.31,1.02]$ & $0.51[0.27,0.94]$ \\
\hline \multirow[t]{2}{*}{ Proteinuria } & Negative & & Ref. \\
\hline & Positive & $5.86[3.35,10.65]$ & $2.85[1.48,5.55]$ \\
\hline \multirow[t]{2}{*}{ AKI } & No & 1 & 1 \\
\hline & Yes & $4.22[2.43,7.48]$ & $1.81[0.92,3.54]$ \\
\hline \multirow[t]{2}{*}{ HTN } & No & 1 & 1 \\
\hline & Yes & $4.65[2.38,9.75]$ & $2.31[1.03,5.56]$ \\
\hline \multirow[t]{2}{*}{ DR } & No & 1 & 1 \\
\hline & Yes & $1.84[1.08,3.09]$ & $0.93[0.52,1.65]$ \\
\hline \multirow[t]{2}{*}{ CVD } & No & 1 & 1 \\
\hline & Yes & $1.18[0.65,2.08]$ & $0.55[0.29,1.96]$ \\
\hline \multirow[t]{3}{*}{ BMI } & $<18.5$ & 1 & 1 \\
\hline & $18.5-24.9$ & $0.85[0.20,5.26]$ & $0.55[0.12,3.62]$ \\
\hline & $>=25$ & $5.17[1.33,30.57]$ & $1.80[0.42,11.51]$ \\
\hline \multirow[t]{2}{*}{$\mathrm{HDL}$} & $>=40$ & 1 & 1 \\
\hline & $<40$ & $4.71[2.72,8.40]$ & $3.19[1.73,5.98]$ \\
\hline \multirow[t]{2}{*}{ LDL } & $<100$ & 1 & 1 \\
\hline & $>=100$ & $3.93[2.17,7.35]$ & $1.29[0.69,2.49]$ \\
\hline \multicolumn{4}{|c|}{ Association parameter (lag of 4 months) } \\
\hline \multicolumn{3}{|c|}{ Assoct (lagged value) } & $4.23[2.53,8.23]$ \\
\hline \multicolumn{3}{|c|}{ AssoctE (Rate of change) } & $1.39[0.11,16.60]$ \\
\hline
\end{tabular}


Table 3

Longitudinal sub-models for DM patients on treatment at Jimma university medical center, Southwest Ethiopia, 2012 to 2020

\begin{tabular}{|c|c|c|}
\hline Fixed effects & Betas & $95 \% \mathrm{Crl}$ \\
\hline Intercept & 4.90 & {$[4.60,5.19]$} \\
\hline ns (Time, 2) 1 & -0.145 & {$[-0.22,-0.07]$} \\
\hline ns (Time, 2)2 & 0.053 & {$[-0.04,0.15]$} \\
\hline weight & 0.002 & {$[-0.002,0.01]$} \\
\hline \multicolumn{3}{|l|}{ Residence } \\
\hline Urban & Ref. & Ref. \\
\hline Rural & 0.02 & {$[-0.12,0.14]$} \\
\hline \multicolumn{3}{|l|}{ Type of treatment } \\
\hline $\mathrm{OHA}$ & Ref. & Ref. \\
\hline Insulin & 0.06 & {$[-0.09,0.25]$} \\
\hline $\mathrm{OHA}+$ Insulin & 0.10 & {$[-0.07,0.28]$} \\
\hline \multicolumn{3}{|l|}{ Variance component } \\
\hline SD of Intercept & & 0.615 \\
\hline SD of ns $($ Time,2) 1 & & 0.593 \\
\hline SD of ns (Time,2)2 & & 0.744 \\
\hline SD of Residual & & 0.346 \\
\hline Corr (Intercept \& Slope) & & -0.012 \\
\hline
\end{tabular}

Figures 


\section{Overall KM survival estimate for DM patients}

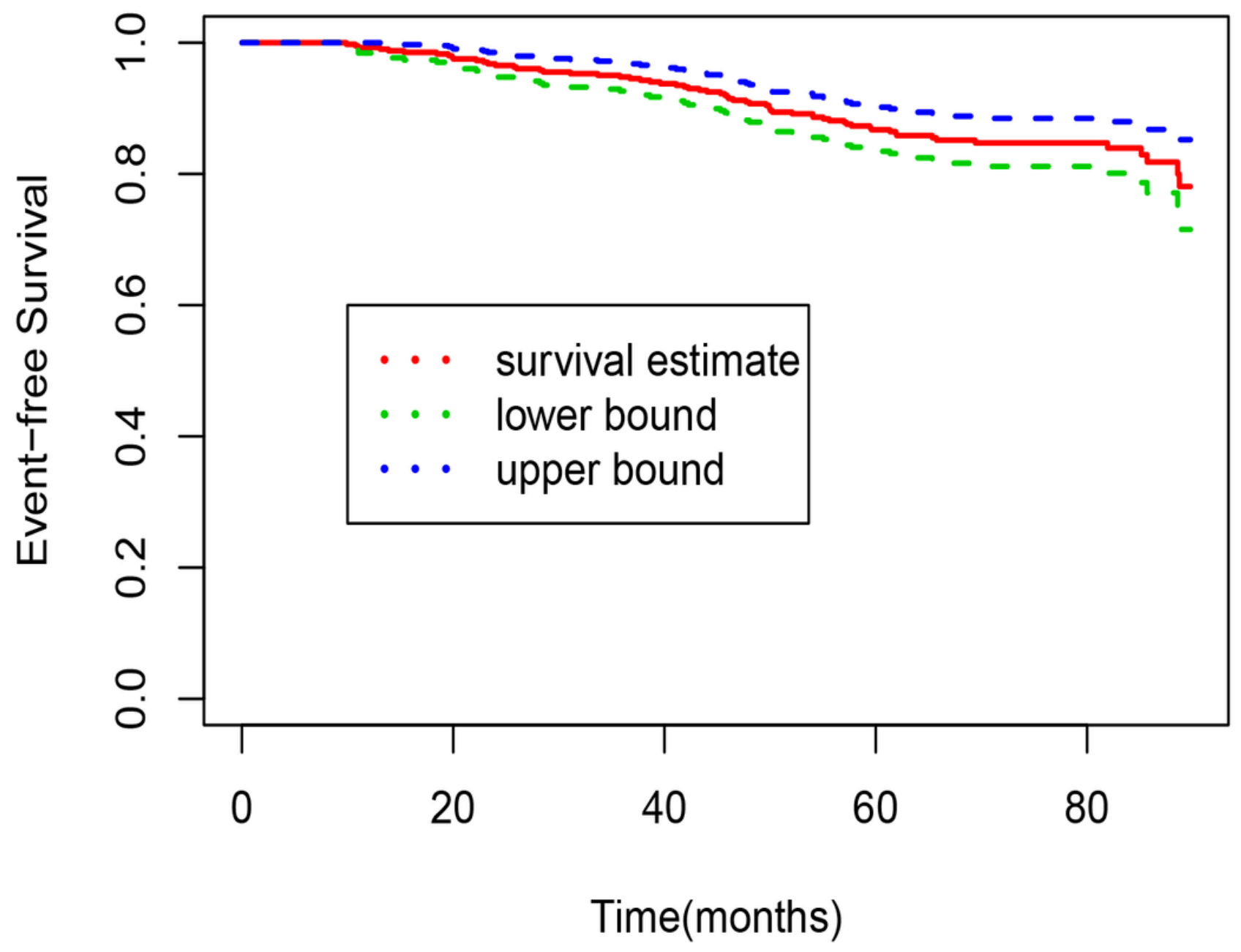

Figure 1

Overall Kaplan Meier of survival curves for DM patients on treatment at Jimma university medical center, Southwest Ethiopia, 2012 to 2020 


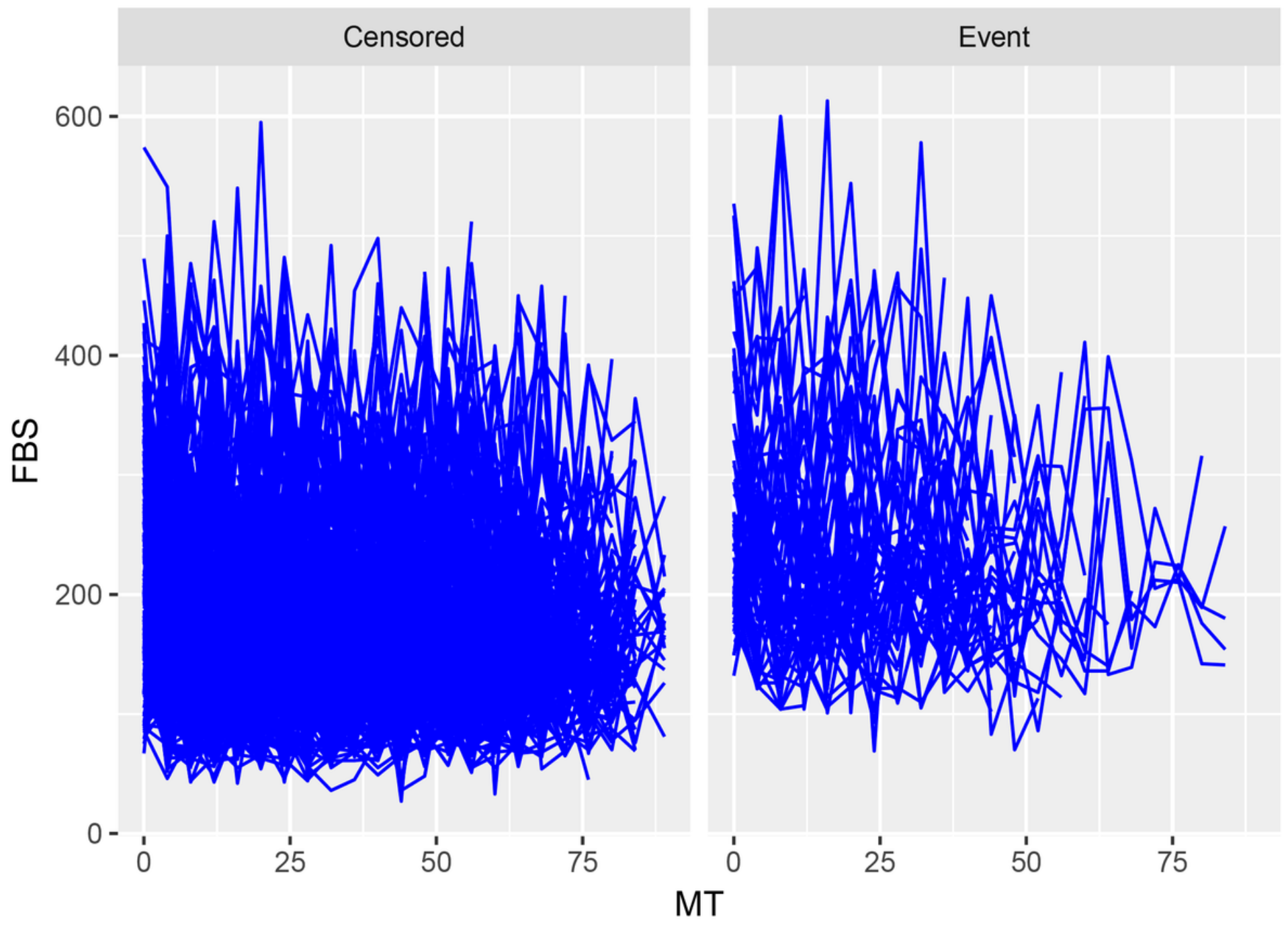

Figure 2

Individual profile plots by status for DM patients on treatment at Jimma university medical center, Southwest Ethiopia, 2012 to 2020. 


\section{mean profile plot by Status}

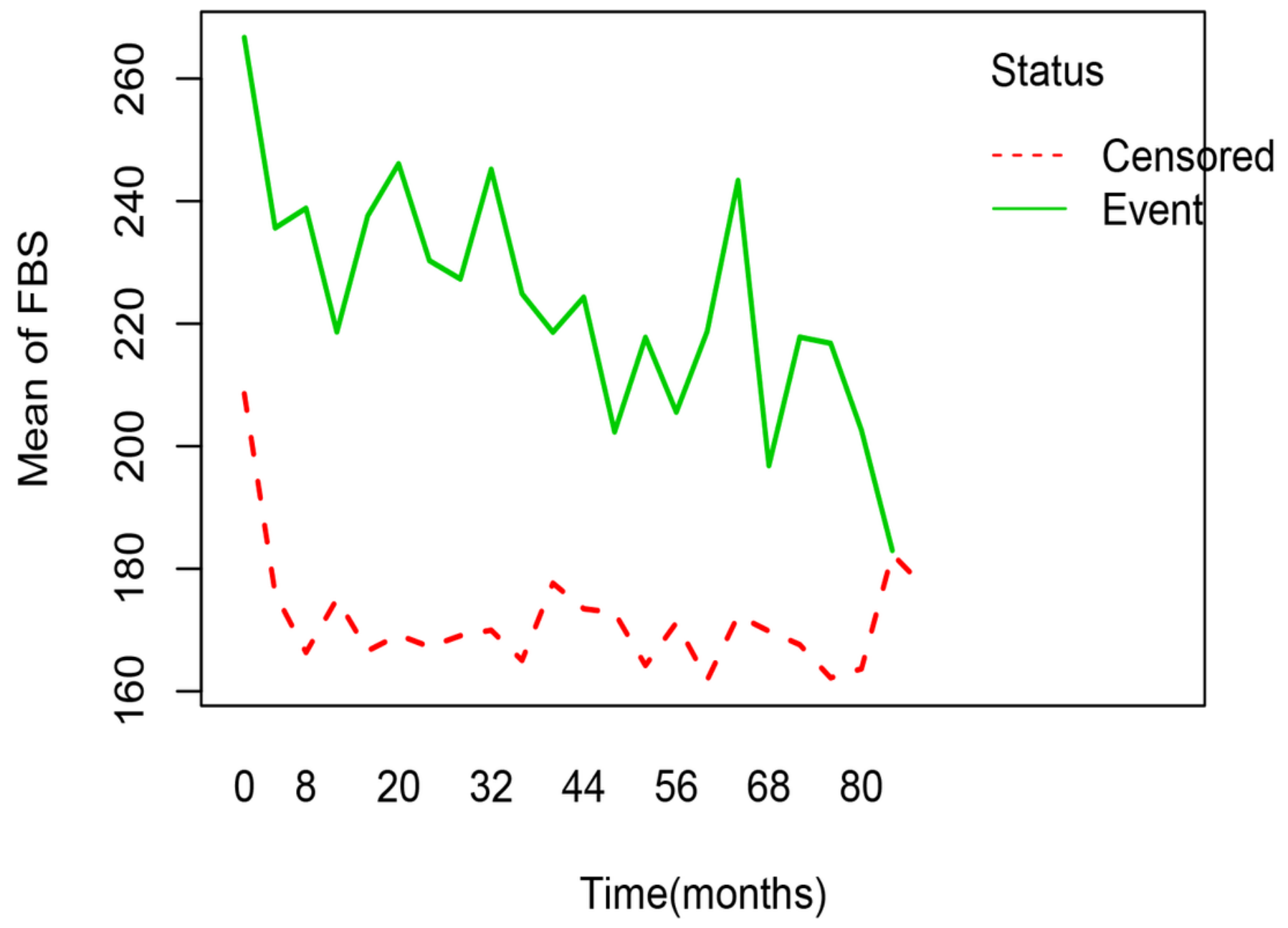

Figure 3

Mean profile of FBS by survival status for DM patients on treatment at Jimma university medical center, Southwest Ethiopia, 2012 to 2020. 


\section{Longitudinal part}

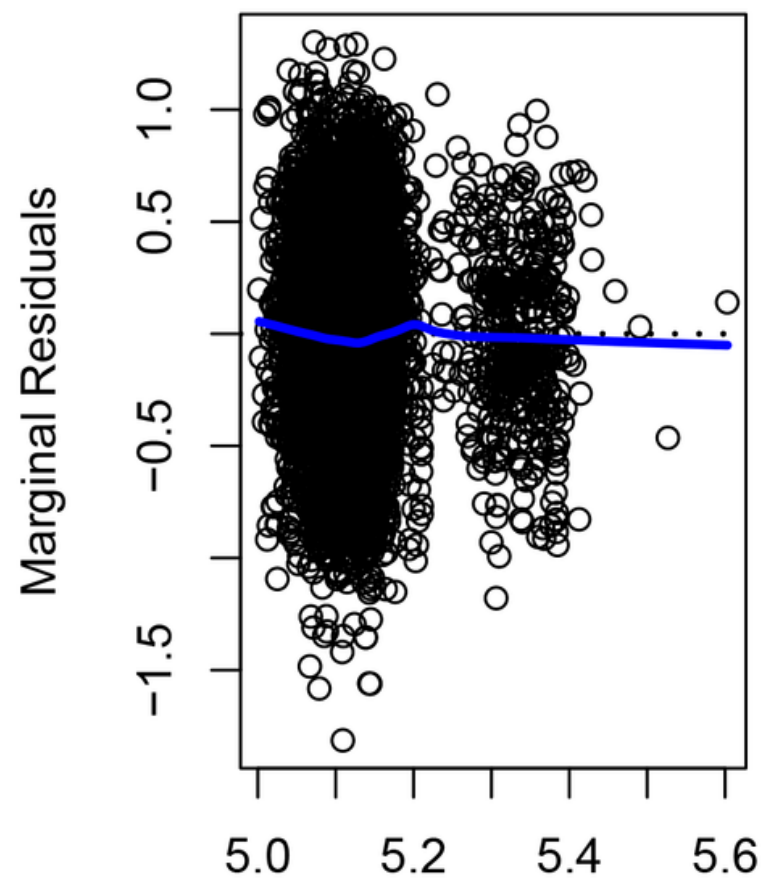

Fitted Values

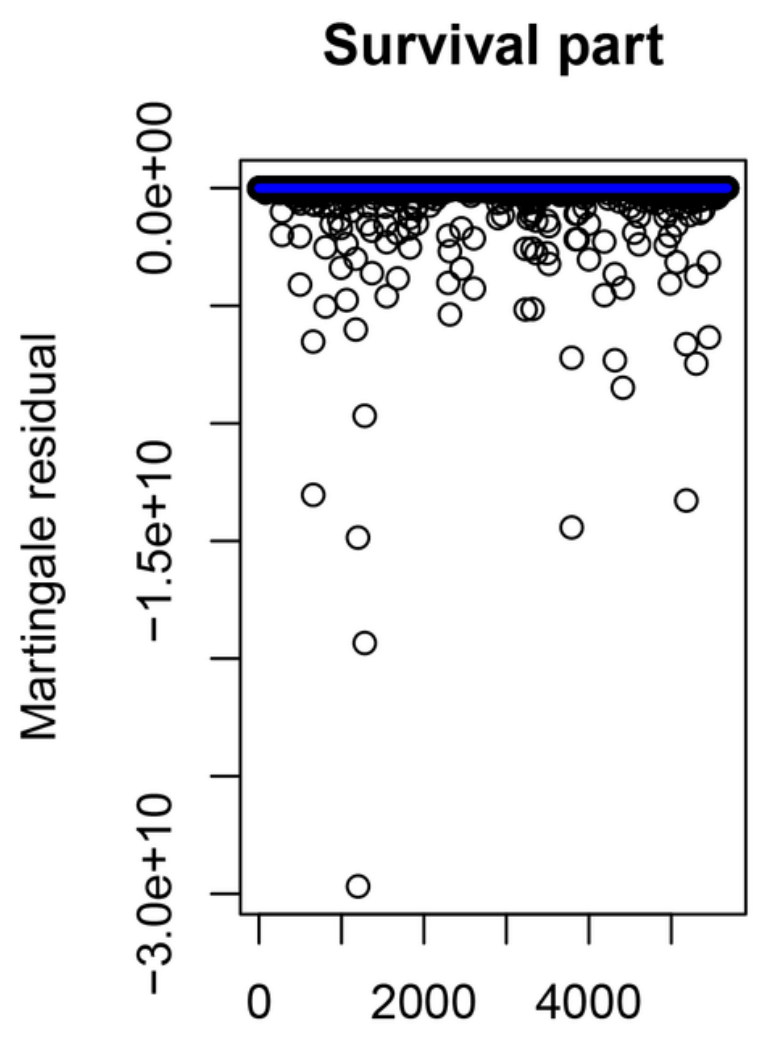

Fitted values

\section{Figure 4}

Marginal and martingale residuals versus the fitted values (goodness of fitness of Bayesian joint modeling) 